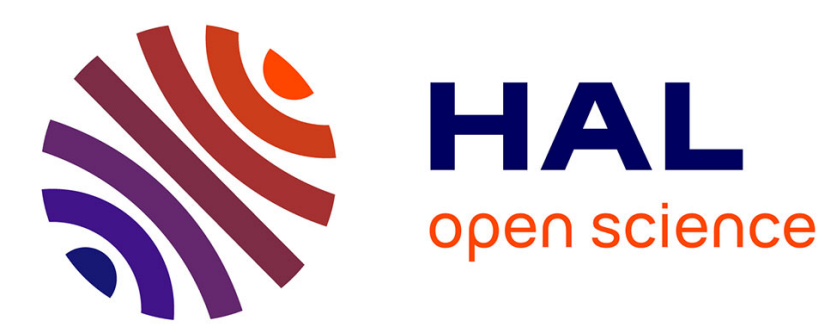

\title{
Homogenization of a one-dimensional diffusion - discrete absorption equation with feedback
}

\author{
Grigory Panasenko, Vitaly Volpert
}

\section{To cite this version:}

Grigory Panasenko, Vitaly Volpert. Homogenization of a one-dimensional diffusion - discrete absorption equation with feedback. Applicable Analysis, 2016, 95, pp.1507 - 1516. 10.1080/00036811.2016.1179288 . hal-01397565

\section{HAL Id: hal-01397565 \\ https://inria.hal.science/hal-01397565}

Submitted on 17 Nov 2016

HAL is a multi-disciplinary open access archive for the deposit and dissemination of scientific research documents, whether they are published or not. The documents may come from teaching and research institutions in France or abroad, or from public or private research centers.
L'archive ouverte pluridisciplinaire HAL, est destinée au dépôt et à la diffusion de documents scientifiques de niveau recherche, publiés ou non, émanant des établissements d'enseignement et de recherche français ou étrangers, des laboratoires publics ou privés. 


\title{
Homogenization of a one-dimensional diffusion - discrete absorption equation with feedback
}

\author{
Grigory Panasenko ${ }^{1}$, Vitaly Volpert ${ }^{2}$ \\ ${ }^{1}$ University of Lyon, UJM-Saint-Etienne, CNRS, Institute Camille Jordan, UMR 5208 \\ SFR MODMAD FED 4169, 42023 Saint-Etienne, France \\ ${ }^{2}$ University of Lyon, UCBL, CNRS, Institute Camille Jordan, UMR 5208 \\ 69622 Villerubanne, France \\ grigory.panasenko@univ-st-etienne.fr,volpert@math.univ-lyon1.fr
}

\begin{abstract}
The paper is devoted to the diffusion equation with discrete absorption described by a sum of Dirac $\delta$-functions. Their supports are located at the nodes of some regular grid with the distance between them determined by the integral of solution. This model describes contraction of biological tissue when cells consume some substance influencing their interaction. In the one-dimensional formulation we prove existence of solutions of the discrete problem and their convergence to the solution of the limiting homogenized problem.
\end{abstract}

Keywords: diffusion - discrete absorption equation with feedback, existence of solution, homogenization

AMS subject classification: 35B27, 35Q53, 35C20

\section{Introduction}

Biological tissues can be considered either as continuous media which are described by partial differential equations of continuum mechanics or as an ensemble of cells described by discrete or discrete-continuous models. The first approach allows the application of various methods of analysis of partial differential equations in order to study existence, stability and dynamics of solutions. However it is less appropriate when we need to take into account individual cell properties, cell-cell interaction, adhesion and other processes at the cellular or intracellular levels. In this case, introduction of individual cells can be very advantageous from the modelling point of view [3]. However mathematical analysis of such models is usually impossible. So the question of transition from individual cell models to continuous models represents one of the main challenges in the modelling of biological tissues. Rather often continuous models of biological fluid or solid mechanics are used without justification 
though biological media are very complex, and in many cases they cannot be described by conventional models like Navier-Stokes equations or elasticity equations.

In this work we introduce and study a model problem where it is possible to justify the transition from the individual cell model to a continuous model with a homogenization technique. It is a nonlinear model where cells regulate the distance between them by means of a chemical substance diffusing in the extracellular matrix and consumed by cells. Such problems arise in various biological applications related to tissue contraction. This is a common physiological property that occurs for many types of tissues under various conditions, in particular for endothelial tissue under inflammation [2], in the process of embryogenesis [11], or in wound healing [7].

Consider diffusion process in the interval $(-R, R)$ containing an array of cells absorbing some substance. This array is under a tensile strain and it is assumed to become stiffer as the total quantity of the absorbed feeding substance increases, i.e. the integral quantity of the absorbed substance modifies the Young modulus of this one-dimensional tissue. This leads to the absorption sites becoming closer together as the domain contracts its length, while the same tensile force $\Phi$ applied to the chain generates smaller displacements of the "saturated cells" than in the case of the "non-saturated cells". In order to simplify the model we assume that the left end of the cell grid is fixed at the point $x=0$ while the tensile force is applied at the right end. Assuming that the distance between the centers of two neighboring cells in "equilibrium", i.e. in abscence of the feeding substance, is a given small parameter, we construct an asymptotic approximation of the model that is a continuous setting. We prove an estimate between the exact and the asymptotic solutions, previously justifying their existence. A linear problem of this type was considered in [14]; however the feedback was not considered there while it is an important part of the process making the problem nonlinear. Various passages from the discrete models to their continuous limits in different contexts were considered in [1]-[13], [16]-[19]. Namely, [6], [8], [16] considered continuous models derived from the atomistic mechanics. Mathematically rigorous derivations were considered in [4], [5], [12], [17]-[19]; these papers used the homogenization technique. A finite element approach to the discrete systems is considered in [16], [19].

Given $R>0$, consider a non-linear boundary value problem for the diffusion - discrete absorption equation on the interval $(-R, R)$ : find a function $u$ defined on the interval $(-R, R)$ such that

$$
\left\{\begin{array}{l}
u^{\prime \prime}(x)=\alpha h \sum_{j=0}^{K} \delta\left(x-Y_{j}^{u}\right) u(x)+f(x), \quad x \in(-R, R), \\
u^{\prime}(-R)=0, \quad u(R)=0, \\
Y_{j}^{u}=j h E_{0}(1+q F[u])^{-1}, \quad j=0, \ldots, K, \\
F[u]=\int_{0}^{E_{0}} u(x) d x,
\end{array}\right.
$$

where the constant coefficients satisfy the following inequalities: $\alpha, q \geq 0, h>0,0<E_{0}<$ $R / 2, K$ is a positive integer, and $h=1 /(K+1)$, the right hand side $f \in C([-R, R])$. Here 


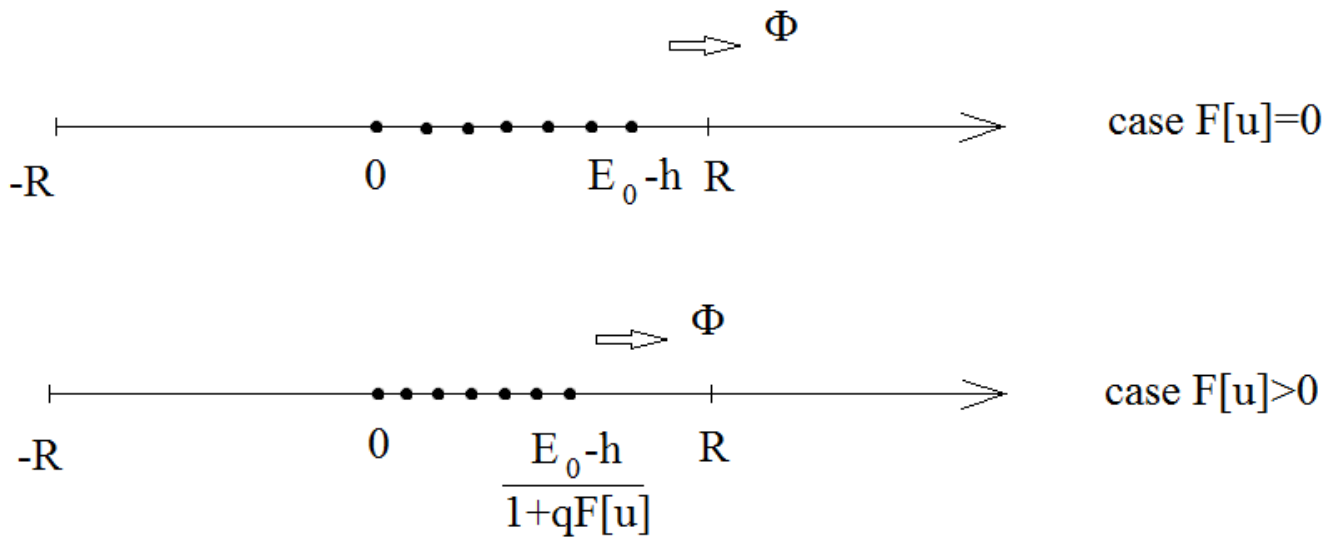

Figure 1: Schematic representation of the model (see the explanation in the text).

$u(x)$ is a concentration of the feeding substance at the point $x$. The discrete absorption sites (cells) form a uniform grid with coordinates $\left\{Y_{j}^{u}, \quad j=0, \ldots, K\right\}$ (Figure 1). The tensile force $\Phi$ is applied at the right end of the grid while its left end is fixed at the point $x=0$. The diffusion process is considered in the interval $(-R, R)$, while the absorption sites occupy only a part of this area: in absence of the feeding substance, the length of the area occupied by cells is $E_{0}$ (although the coordinate of the last cell of the chain is $E_{0}-h$ ). The integral quantity of the substance $F[u]$ in interval $\left(0, E_{0}\right)$ influences the distance between the centers of the cells: the larger this integral is, more contracted the grid is. Namely, its length is equal to $E_{0}(1+q F[u])^{-1}$. The coordinates of the sites are supposed to satisfy a standard elastic scalar springs model [6] where the ratio of the Young's moduli of the "springs" in the saturated state and in the case of absence of the feeding substance is equal to $1+q F[u]$, where $q$ is the stiffening coefficient: it characterizes the increasing of the Young's modulus as $F[u]$ increases, $\alpha h$ is the ratio of the discrete absorption and diffusivity. We look for a bounded solution for which the denominator in $(1.1)_{3}$ is positive.

Below we prove the existence of a solution of this problem and justify the passage to the non-linear continuous model. The simplified model problems allow us to study these questions rigorously.

Let us rewrite equations $(1.1)_{1,2}$ in the form: find $u \in C([-R, R])$ such that

$$
u(x)=-\int_{x}^{R}\left(\Lambda u(t)+\int_{-R}^{t} f(\theta) d \theta\right) d t, \quad x \in[-R, R],
$$

where

$$
\Lambda u(x)=h \alpha \int_{-R}^{x} \sum_{j=0}^{K} \delta\left(\theta-Y_{j}^{u}\right) u(\theta) d \theta
$$

that is 


$$
\Lambda u(x)=h \alpha \sum_{j: Y_{j}^{u} \leq x} u\left(Y_{j}^{u}\right) .
$$

In section 2 applying the Shauder's fixed point theorem we prove the existence of a solution in the ball $B=\left\{v \in C([-R, R]), \quad\|v\|_{C} \leq \rho\right\}, \rho=8 R^{2}\|f\|_{C}$ for sufficiently small $\alpha$ and $q$.

In section 3 we introduce the homogenized continuous model

$$
\left\{\begin{array}{l}
\hat{u}^{\prime \prime}(x)=\alpha \frac{1+q F[\hat{u}]}{E_{0}} \chi_{[0, Y \hat{u}]}(x) \hat{u}(x)+f(x), \quad x \in(-R, R), \\
\hat{u}^{\prime}(-R)=0, \quad \hat{u}(R)=0,
\end{array}\right.
$$

where $Y^{\hat{u}}=E_{0}(1+q F[\hat{u}])^{-1}$ and $\chi_{E}$ is a characteristic function of the set $E$. This model may be derived heurictically by averaging the absorption sites within the absorption area (its length is approximatevely equal to $Y^{\hat{u}}$ ). The existence and uniqueness of a solution to the homogenized problem in the ball $B$ is proved using a Banach's fixed point theorem.

Finally, in section 3 an error estimate is proved for the difference of the solutions to problems (1.1) and (1.4). It is of order $h$, so that the initial and the homogenized models are $O(h)$-close.

\section{Existence of solution to the diffusion-discrete ab- sorption equation with feedback}

In the present section we prove the existence of the solution (1.2) for "not too large" coefficients $\alpha$ and $q$. The proof is based on the Schauder's fixed point theorem. Set $\alpha^{*}=1 /(4 R)$ and

$$
q^{*}=\left\{\begin{array}{cl}
1 /\left(16 E_{0} R^{2}\|f\|_{C}\right) & , \quad\|f\|_{C} \neq 0 \\
\infty & , \quad\|f\|_{C}=0
\end{array}\right.
$$

Theorem 1. For $\alpha \in\left[0, \alpha^{*}\right), q \in\left[0, q^{*}\right)$ there exists a function $u \in B$, satisfying (1.2).

Proof. Let $\alpha$ be positive (case $\alpha=0$ is trivial). For any $q \in\left[0, q^{*}\right)$ and $v \in B$, the following estimate holds:

$$
|F[v]| \leq E_{0}\|v\|_{C} \leq E_{0} \rho=8 E_{0} R^{2}\|f\|_{C}<\frac{1}{q^{*}}<\frac{1}{q}
$$

Therefore the denominator in $(1.1)_{3}$ is positive. Consider the operator $T: B \rightarrow C([-R, R])$ such that for any $v \in B$,

$$
(T v)(x)=-\int_{x}^{R}\left(\Lambda v(t)+\int_{-R}^{t} f(\theta) d \theta\right) d t .
$$

Evidently, $T v$ is a piecewise differentiable continuous function, such that for any $v \in B$ 


$$
\left\{\begin{array}{l}
\|T v\|_{C} \leq 2 R\left(\alpha\|v\|_{C}+2 R\|f\|_{C}\right) \\
\left\|(T v)^{\prime}\right\|_{L^{\infty}} \leq \alpha\|v\|_{C}+2 R\|f\|_{C}
\end{array}\right.
$$

and for $\alpha \in\left[0, \alpha^{*}\right),\|T v\|_{C} \leq 2 R \alpha \rho+4 R^{2}\|f\|_{C}<\rho$, so that $T B \subset B$.

In the same way we get that if $\beta=\left\{v \in B \cap W^{1, \infty} \mid\left\|v^{\prime}\right\|_{L^{\infty}} \leq \frac{\rho}{2 R}\right\}$ then $T \beta \subset \beta$. Evidently, $\beta$ is convex and being bounded in $W^{1, \infty}$ it is compact in $C$. Further we will apply the Shauder's fixed point theorem to this set $\beta$.

Moreover, for any $v \in B$, the following estimate holds:

$$
|F[v]| \leq E_{0}\|v\|_{C} \leq E_{0} \rho
$$

and for all $v_{1}, v_{2} \in B$,

$$
\begin{gathered}
\left|\left(1+q F\left[v_{1}\right]\right)^{-1}-\left(1+q F\left[v_{2}\right]\right)^{-1}\right| \leq \max _{\xi:|\xi| \leq E_{0} \rho} \frac{q}{(1+q \xi)^{2}}\left|F\left[v_{1}\right]-F\left[v_{2}\right]\right| \leq \\
\leq \frac{q}{\left(1-q E_{0} \rho\right)^{2}} E_{0}\left\|v_{1}-v_{2}\right\|_{C} .
\end{gathered}
$$

Taking into account that $q E_{0} \rho \leq \frac{1}{2}$ for all $q \leq q^{*}$, we get

$$
\left|\left(1+q F\left[v_{1}\right]\right)^{-1}-\left(1+q F\left[v_{2}\right]\right)^{-1}\right| \leq 4 q E_{0}\left\|v_{1}-v_{2}\right\|_{C} .
$$

Let us estimate the difference $Y_{j}^{v_{1}}-Y_{j}^{v_{2}}$ :

$$
\left|Y_{j}^{v_{1}}-Y_{j}^{v_{2}}\right|=j h E_{0}\left|\left(1+q F\left[v_{1}\right]\right)^{-1}-\left(1+q F\left[v_{2}\right]\right)^{-1}\right| \leq K h E_{0}\left(4 q E_{0}\right)\left\|v_{1}-v_{2}\right\|_{C} .
$$

Hence

$$
\left|Y_{j}^{v_{1}}-Y_{j}^{v_{2}}\right| \leq 4 q E_{0}^{2}\left\|v_{1}-v_{2}\right\|_{C} .
$$

Next, we prove that $T$ is continuous on $B$. Let $\left\{u_{n}\right\}$ be a sequence in $B$ converging to $u \in B$. Let $\varepsilon>0$. Since $u$ is uniformly continuous, then there exist $\delta>0$ such that for any $x^{\prime}, x^{\prime \prime} \in[-R, R]$ satisfying $\left|x^{\prime}-x^{\prime \prime}\right| \leq \delta$, we get:

$$
\left|u\left(x^{\prime}\right)-u\left(x^{\prime \prime}\right)\right|<\frac{\varepsilon}{4 \alpha R} .
$$

So, there exist an integer $N$ such that, for all $n \geq N$,

$$
\left\|u_{n}-u\right\|_{C}<\min \left\{\frac{\varepsilon}{4\left(R \alpha+2 q \alpha E_{0}^{2} \rho\right)}, \frac{\delta}{4 E_{0}^{2} q}, \frac{\varepsilon}{2 \alpha}\right\}
$$

Denote by $j_{v}(t)$ the integer part of the fraction $\frac{t(1+q F[v])}{h E_{0}}$ :

$$
j_{v}(t)=\left[\frac{t(1+q F[v])}{h E_{0}}\right], \quad t>0,
$$

i.e. the greatest $j$ such that $Y_{j}^{v} \leq t$. Evidently, 


$$
\left|\frac{t\left(1+q F\left[u_{n}\right]\right)}{h E_{0}}-\frac{t(1+q F[u])}{h E_{0}}\right|=\frac{t q}{h E_{0}}\left|F\left[u_{n}\right]-F[u]\right| \leq \frac{t q}{h}\left\|u_{n}-u\right\|_{C} \leq \frac{t q \varepsilon}{2 h \alpha} .
$$

Therefore for sufficiently small $\varepsilon$,

$$
\left|j_{u_{n}}(t)-j_{u}(t)\right| \leq 1
$$

i.e. the number of terms in the sums (1.3) for $u_{n}$ and $u$ is either the same or there is only one supplementary term in one of these sums. Let this term be $u_{n}\left(Y_{j_{u_{n}}(t)}^{u_{n}}\right)$ (the case when this supplementary term is $u\left(Y_{j_{u}(t)}^{u}\right)$ is considered in the same way). This difference of number of terms exists only for the values of $t$ between $Y_{j}^{u_{n}}$ and $Y_{j}^{u}\left(\operatorname{here} j_{u_{n}}(t)=j_{u}(t)+1\right)$, i.e. on the interval of the length (see (2.5))

$$
\Delta \leq \max _{0 \leq j \leq K}\left|Y_{j}^{u_{n}}-Y_{j}^{u}\right| \leq 4 q E_{0}^{2}\left\|u_{n}-u\right\|_{C} .
$$

Then we get:

$\left|T u_{n}(x)-T u(x)\right|=\left|\int_{x}^{R}\left(\Lambda u_{n}(t)-\Lambda u(t)\right) d t\right|=h \alpha\left|\int_{x}^{R}\left(\sum_{j=0}^{j_{u_{n}}(t)} u_{n}\left(Y_{j}^{u_{n}}\right)-\sum_{j=0}^{j_{u}(t)} u\left(Y_{j}^{u}\right)\right) d t\right| \leq$

$\leq 2 R \alpha \max _{0 \leq j \leq K}\left|u_{n}\left(Y_{j}^{u_{n}}\right)-u\left(Y_{j}^{u}\right)\right|+\alpha \Delta \rho \leq 2 R \alpha \max _{0 \leq j \leq K}\left|u_{n}\left(Y_{j}^{u_{n}}\right)-u\left(Y_{j}^{u}\right)\right|+4 q E_{0}^{2} \alpha\left\|u_{n}-u\right\|_{C} \rho$.

Therefore since

$$
\left|Y_{j}^{u_{n}}-Y_{j}^{u}\right| \leq 4 q E_{0}^{2}\left\|u_{n}-u\right\|_{C} \leq 4 q E_{0}^{2} \frac{\delta}{4 E_{0}^{2} q}=\delta
$$

we get

$$
\left|T u_{n}(x)-T u(x)\right| \leq 2 R \alpha\left(\left\|u_{n}-u\right\|_{C}+\frac{\varepsilon}{4 \alpha R}\right)+4 q E_{0}^{2} \alpha\left\|u_{n}-u\right\|_{C} \rho,
$$

by virtue of the estimate

$$
\left|Y_{j}^{u_{n}}-Y_{j}^{u}\right| \leq 4 q E_{0}^{2}\left\|u_{n}-u\right\|_{C} \leq 4 q E_{0}^{2} \frac{\delta}{4 E_{0}^{2} q}=\delta .
$$

Taking into account that for all $n \geq N$

$$
\left(2 R \alpha+4 q E_{0}^{2} \alpha \rho\right)\left\|u_{n}-u\right\|_{C} \leq \frac{\varepsilon}{2}
$$

(see (2.7)) we get $\left|T u_{n}(x)-T u(x)\right| \leq \varepsilon$, that is $\left\|T u_{n}-T u\right\|_{C} \leq \varepsilon$ for all $n \geq N$. This means that the sequence $\left\{T u_{n}\right\}$ converges to $T u$ in $C$. Hence the operator $T$ is continuous. 
Applying now the Schauder fixed point theorem [10] to the convex and compact set $\beta$, we get the existence of a solution. The theorem is proved.

Remark 1. Applying estimates (2.2) for the fixed point $u$, we get:

$$
\left\{\begin{array}{l}
\|u\|_{C} \leq 8 R^{2}\|f\|_{C} \\
\left\|(u)^{\prime}\right\|_{L^{\infty}} \leq\left(8 \alpha R^{2}+2 R\right)\|f\|_{C},
\end{array}\right.
$$

\section{Existence of solution of the homogenized equation}

In the present section we introduce the homogenized equation: a non-linear diffusion-absorption equation with the absorption coefficient depending on the solution. We prove the existence and uniqueness of a solution. Consider the homogenized problem

$$
\left\{\begin{array}{l}
\hat{u}^{\prime \prime}(x)=\alpha \frac{1+q F[\hat{u}]}{E_{0}} \chi_{[0, Y \hat{u}]}(x) \hat{u}(x)+f(x), \quad x \in(-R, R), \\
\hat{u}^{\prime}(-R)=0, \quad \hat{u}(R)=0 .
\end{array}\right.
$$

where $Y^{\hat{u}}=E_{0}(1+q F[\hat{u}])^{-1}$.

We have

$$
\hat{u}(x)=-\int_{x}^{R}\left(\hat{\Lambda} \hat{u}(t)+\int_{-R}^{t} f(\theta) d \theta\right) d t, \quad x \in[-R, R],
$$

where

$$
\hat{\Lambda} v(t)=\alpha \int_{0}^{t} \chi_{\left[0, Y^{v}\right]}(\theta) \frac{1+q F[v]}{E_{0}} v(\theta) d \theta
$$

i.e. for $t \geq 0$,

$$
\hat{\Lambda} v(t)=\alpha \frac{1+q F[v]}{E_{0}} \int_{0}^{\min \left\{t, Y^{v}\right\}} v(\theta) d \theta
$$

while for $t<0, \hat{\Lambda} v(t)=0$.

Set

$$
\alpha^{* *}=\min \left\{\frac{E_{0}}{2 R\left(1+2 q E_{0} \rho\right)\left(R+8 q E_{0}^{2} \rho\right)}, \frac{E_{0}}{4 R^{2}\left(1+q E_{0} \rho\right)}\right\} .
$$

Theorem 2. For $\alpha \in\left[0, \alpha^{* *}\right)$ there exists a unique function $\hat{u} \in C([-R, R])$, satisfying (3.2) and belonging to the ball $B=\left\{v \in C([-R, R]), \quad\|v\|_{C} \leq \rho\right\}$.

Proof. We have the following estimates

$$
|\hat{\Lambda} v(t)| \leq \alpha R \frac{1+q E_{0}\|v\|_{C}}{E_{0}}\|v\|_{C},
$$




$$
\begin{gathered}
\left|\hat{\Lambda} v_{1}(t)-\hat{\Lambda} v_{2}(t)\right|=\frac{\alpha}{E_{0}}\left|\left(1+q F\left[v_{1}\right]\right) \int_{0}^{t}\left(v_{1}(\theta)-v_{2}(\theta)\right) d \theta+q\left(F\left[v_{1}\right]-F\left[v_{2}\right]\right) \int_{0}^{t} v_{2}(\theta) d \theta\right| \leq \\
\leq \frac{\alpha}{E_{0}}\left(\left(1+q E_{0}\left\|v_{1}\right\|_{C}\right) R\left\|v_{1}-v_{2}\right\|_{C}+q E_{0}\left\|v_{1}-v_{2}\right\|_{C} R\left\|v_{2}\right\|_{C}\right) .
\end{gathered}
$$

Therefore if $t \leq \min \left\{Y^{v_{1}}, Y^{v_{2}}\right\}$, then

$$
\left|\hat{\Lambda} v_{1}(t)-\hat{\Lambda} v_{2}(t)\right| \leq \frac{\alpha R}{E_{0}}\left(1+q E_{0}\left(\left\|v_{1}\right\|_{C}+\left\|v_{2}\right\|_{C}\right)\right)\left\|v_{1}-v_{2}\right\|_{C}
$$

If $t>\min \left\{Y^{v_{1}}, Y^{v_{2}}\right\}$, then we have to add a supplementary term in the upper bound. Without loss of generality we can assume that $Y^{v_{1}}<Y^{v_{2}}$. Then this supplementary term can be written in the form:

$$
\frac{\alpha}{E_{0}}\left(1+q\left|F\left[v_{2}\right]\right|\right) \int_{Y^{v_{1}}}^{Y^{v_{2}}}\left|v_{2}(\theta)\right| d \theta
$$

This expression is less than the following one:

$$
\frac{\alpha}{E_{0}}\left(1+q E_{0}\left\|v_{2}\right\|_{C}\right)\left\|v_{2}\right\|_{C}\left(Y^{v_{2}}-Y^{v_{1}}\right)
$$

and can be estimated by

$$
\frac{\alpha}{E_{0}}\left(1+q E_{0}\left\|v_{2}\right\|_{C}\right)\left\|v_{2}\right\|_{C} 4 q E_{0}^{2}\left\|v_{1}-v_{2}\right\|_{C}
$$

(see $(2.5))$. Finally, we get

$$
\left|\hat{\Lambda} v_{1}(t)-\hat{\Lambda} v_{2}(t)\right| \leq \frac{\alpha}{E_{0}}\left(1+q E_{0}\left(\left\|v_{1}\right\|_{C}+\left\|v_{2}\right\|_{C}\right)\right)\left(R+4 q E_{0}^{2}\left(\left\|v_{1}\right\|_{C}+\left\|v_{2}\right\|_{C}\right)\right)\left\|v_{1}-v_{2}\right\|_{C}
$$

Consider the operator $\hat{T}: B \rightarrow C([-R, R])$ such that for any $v \in B$,

$$
(\hat{T} v)(x)=-\int_{x}^{R}\left(\hat{\Lambda} v(t)+\int_{-R}^{t} f(\theta) d \theta\right) d t
$$

Next, for any $v_{1}, v_{2} \in B$, the following estimate holds:

$$
\left\|\hat{T} v_{1}-\hat{T} v_{2}\right\|_{C} \leq 2 R\left\|\hat{\Lambda} v_{1}-\hat{\Lambda} v_{2}\right\|_{C} \leq \omega\left\|v_{1}-v_{2}\right\|_{C},
$$

where

$$
\omega=\frac{2 \alpha R}{E_{0}}\left(1+2 q E_{0} \rho\right)\left(R+8 q E_{0}^{2} \rho\right) .
$$

The inequality $\alpha<\alpha^{* *}$ yields $\omega<1$.

Let us check that $\hat{T} B \subset B$. Indeed, 


$$
\|\hat{T} v\|_{C} \leq 2 R\|\hat{\Lambda} v\|_{C}+4 R^{2}\|f\|_{C} \leq \frac{2 \alpha R^{2}}{E_{0}}\left(1+q E_{0} \rho\right) \rho+\frac{\rho}{2} \leq \rho .
$$

Taking into account that $\hat{T} B \subset B$, we see that $\hat{T}$ is a contracting operator in $B$ and so by the Banach fixed point theorem there exist a unique fixed point in $B$. The theorem is proved.

Remark 2. Applying estimates (3.5) for the fixed point $\hat{u}$, we get:

$$
\left\|\hat{u}^{\prime}\right\|_{C} \leq\|\hat{\Lambda} \hat{u}\|_{C}+2 R\|f\|_{C} .
$$

Hence

$$
\left\|\hat{u}^{\prime}\right\|_{C} \leq \frac{\alpha}{E_{0}}\left(1+q E_{0}\|\hat{u}\|_{C}\right) R\|\hat{u}\|_{C}+2 R\|f\|_{C}
$$

and

$$
\left\|\hat{u}^{\prime}\right\|_{C} \leq\left(\frac{8 \alpha R^{3}}{E_{0}}\left(1+8 q E_{0} R^{2}\|f\|_{C}\right)+2 R\right)\|f\|_{C} .
$$

\section{Error estimate for the discrete and continuous mod- els}

We will prove in this section that the solution of the discrete problem is close to the solution of the continuous problem. Compare the solutions to equations (1.2) and (3.2). Subtracting these equations we get the following equation for the difference $w=u-\hat{u}$ :

$$
w(x)=-\int_{x}^{R}(\Lambda u(t)-\hat{\Lambda} \hat{u}(t)) d t .
$$

Then

$$
w(x)=-\left\{\left.\int_{x}^{R} \Lambda\right|_{Y_{j}^{u}} w(t) d t+\int_{x}^{R}\left(\left.\Lambda\right|_{Y_{j}^{u}} \hat{u}(t)-\hat{\Lambda} \hat{u}(t)\right) d t\right\}
$$

where

$$
\left.\Lambda\right|_{Y_{j}^{u}} w(t)=h \alpha \sum_{j: Y_{j}^{u} \leq t} w\left(Y_{j}^{u}\right)
$$

We have the estimate:

$$
|\Lambda|_{Y_{j}^{u}} w(t) \mid \leq \alpha\|w\|_{C}
$$

The second integral in (4.2) equals the following one:

$$
\int_{x}^{R}\left(\left.\Lambda\right|_{Y_{j}^{u}} \hat{u}(t)-\left.\Lambda\right|_{Y_{j}^{\hat{u}}} \hat{u}(t)\right) d t+\int_{x}^{R}\left(\left.\Lambda\right|_{Y_{j}^{\hat{u}}} \hat{u}(t)-\hat{\Lambda} \hat{u}(t)\right) d t .
$$

Let us estimate these two terms. 
a) We have

$$
\left.\Lambda\right|_{Y_{j}^{u}} \hat{u}(t)-\left.\Lambda\right|_{Y_{j}^{\hat{u}}} \hat{u}=h \alpha\left(\sum_{j: Y_{j}^{u} \leq t} \hat{u}\left(Y_{j}^{u}\right)-\sum_{j: Y_{j}^{\hat{u}} \leq t} \hat{u}\left(Y_{j}^{\hat{u}}\right)\right) .
$$

As in the proof of Theorem 1, the difference of number of terms in these sums is $\left|j_{u}(t)-j_{\hat{u}}(t)\right|$ and it can be evaluated as follows:

$$
\left|j_{u}(t)-j_{\hat{u}}(t)\right| \leq \frac{t q}{h}\|u-\hat{u}\|_{C}+1
$$

Therefore

$$
\begin{gathered}
|\Lambda|_{Y_{j}^{u}} \hat{u}(t)-\left.\Lambda\right|_{Y_{j}^{\hat{u}}} \hat{u} \mid \leq h \alpha\left\{\sum_{j=0}^{K}\left|\hat{u}\left(Y_{j}^{u}\right)-\hat{u}\left(Y_{j}^{\hat{u}}\right)\right|+\left(\frac{t q}{h}\|u-\hat{u}\|_{C}+1\right)\|\hat{u}\|_{C}\right\} \leq \\
\leq h \alpha \sum_{j=0}^{K}\left\|\hat{u}^{\prime}\right\|_{L^{\infty}}\left|Y_{j}^{u}-Y_{j}^{\hat{u}}\right|+R \alpha q\|u-\hat{u}\|_{C}\|\hat{u}\|_{C}+h \alpha\|\hat{u}\|_{C} \leq \\
\leq(K+1) h \alpha\left\|\hat{u}^{\prime}\right\|_{L^{\infty}} 4 q E_{0}^{2}\|u-\hat{u}\|_{C}+R \alpha q\|u-\hat{u}\|_{C}\|\hat{u}\|_{C}+h \alpha\|\hat{u}\|_{C} .
\end{gathered}
$$

We applied here the estimate (2.5) for $\left|Y_{j}^{u}-Y_{j}^{\hat{u}}\right|$. Finally, we obtain

$$
\left|\int_{x}^{R}\left(\left.\Lambda\right|_{Y_{j}^{u}} \hat{u}(t)-\left.\Lambda\right|_{Y_{j}^{\hat{u}}} \hat{u}(t)\right) d t\right| \leq 2 R \alpha\left(\left(\left\|\hat{u}^{\prime}\right\|_{L^{\infty}} 4 q E_{0}^{2}+\|\hat{u}\|_{C} R q\right)\|u-\hat{u}\|_{C}+h\|\hat{u}\|_{C}\right)
$$

b) In order to evaluate the second term in (4.5), consider two cases: $\left.b_{1}\right), t \leq Y^{\hat{u}}$ and $b_{2}$ ), $t>Y^{\hat{u}}$

$\left.b_{1}\right)$ For $t \leq Y^{\hat{u}}=\frac{E_{0}}{1+q F[\hat{u}]}$ we get:

$$
\begin{aligned}
& \left.\Lambda\right|_{Y_{j}^{\hat{u}}} \hat{u}(t)=h \alpha \sum_{j: Y_{j}^{\hat{u}} \leq t} \hat{u}\left(Y_{j}^{\hat{u}}\right)=h \alpha \sum_{0 \leq j \leq \frac{(1+q F \hat{u}]) t}{E_{0} h}} \hat{u}\left(j h \frac{E_{0}}{1+q F[\hat{u}]}\right)= \\
& =\alpha \int_{0}^{\frac{(1+q F \hat{u}]) t}{E_{0}}} \hat{u}\left(\theta \frac{E_{0}}{1+q F[\hat{u}]}\right) d \theta+r_{h}=\frac{1+q F[\hat{u}]}{E_{0} h} \alpha \int_{0}^{t} \hat{u}(\bar{\theta}) d \bar{\theta}+r_{h}
\end{aligned}
$$

where

$$
r_{h}=\sum_{0 \leq j \leq\left[\frac{(1+q F[\hat{u}]) t}{E_{0} h}\right]} \int_{j h}^{j h+h} \alpha\left(\hat{u}\left(j h \frac{E_{0}}{1+q F[\hat{u}]}\right)-\hat{u}\left(\theta \frac{E_{0}}{1+q F[\hat{u}]}\right)\right) d \theta+
$$




$$
+\int_{\frac{(1+q F[\hat{u}]) t}{E_{0}}}^{\left[\frac{(1+q F[\hat{u}]) t}{E_{0} h}\right] h+h} \alpha \hat{u}\left(\theta \frac{E_{0}}{1+q F[\hat{u}]}\right) d \theta .
$$

$\left.b_{2}\right)$ For $t>Y^{\hat{u}}=\frac{E_{0}}{1+q F[\hat{u}]}$,

$$
\left.\Lambda\right|_{Y_{j}^{\hat{u}}} \hat{u}(t)=\mathrm{const}=\frac{1+q F[\hat{u}]}{E_{0} h} \alpha \int_{0}^{\frac{E_{0}}{1+q F[\hat{u}]}} \hat{u}(\bar{\theta}) d \bar{\theta}+r_{h},
$$

where

$$
r_{h}=\sum_{0}^{K-1} \int_{j h}^{j h+h} \alpha\left(\hat{u}\left(j h \frac{E_{0}}{1+q F[\hat{u}]}\right)-\hat{u}\left(\theta \frac{E_{0}}{1+q F[\hat{u}]}\right)\right) d \theta
$$

Hence

$$
\left|r_{h}\right| \leq\left(\left\|\hat{u}^{\prime}\right\|_{L^{\infty}} \frac{E_{0}}{1+q F[\hat{u}]}+\|\hat{u}\|_{C}\right) \alpha h .
$$

Applying estimate (3.10) and $|F[\hat{u}]| \leq E_{0} \rho$, we get

$$
\left|r_{h}\right| \leq\left(\left(\frac{8 \alpha R^{3}}{E_{0}}\left(1+8 q E_{0} R^{2}\|f\|_{C}\right)+2 R\right) \frac{E_{0}}{1-8 q E_{0} R^{2}\|f\|_{C}}+8 R^{2}\right) \alpha h\|f\|_{C}
$$

Thus, (4.2) - (4.5), (4.7) and

$$
\left|\int_{x}^{R}\left(\left.\Lambda\right|_{Y_{j}^{\hat{u}}} \hat{u}(t)-\hat{\Lambda} \hat{u}\right) d t\right| \leq R \sup _{t \in[0, R]}\left|r_{h}\right|
$$

yield:

$$
\begin{aligned}
& \|w\|_{C} \leq R \alpha\|w\|_{C}+2 R \alpha\left(\left(\frac{8 \alpha R^{3}}{E_{0}}\left(1+8 q E_{0} R^{2}\|f\|_{C}\right)+2 R\right) 4 q E_{0}^{2}\|f\|_{C}+\right. \\
& \left.+8 R^{3} q\|f\|_{C}\right)\|w\|_{C}+16 R^{3} \alpha h\|f\|_{C}+ \\
& +\left(\left(\frac{8 \alpha R^{3}}{E_{0}}\left(1+8 q E_{0} R^{2}\|f\|_{C}\right)+2 R\right) \frac{E_{0} R}{1-8 q E_{0} R^{2}\|f\|_{C}}+8 R^{2}\right) \alpha h\|f\|_{C} R
\end{aligned}
$$

In order to formulate the main result of this section, set

$$
\alpha^{* * *}=\min \left\{\left(R\left(1+2\left(\frac{4 R^{3}}{E_{0}}\left(1+8 q E_{0} R^{2}\|f\|_{C}\right)+2 R\right)\|f\|_{C} 4 q E_{0}^{2}+8 R^{3} q\|f\|_{C}\right)\right)^{-1}, \frac{1}{2}\right\}
$$

Theorem 3. For $\alpha \in\left[0, \min \left\{\alpha^{*}, \alpha^{* *}, \alpha^{* * *}\right\}\right), \quad q \in\left(0, q^{*}\right)$, there exists a solution $u \in B$ to problem (1.2) and a unique solution $\hat{u} \in B$ to problem (3.2), and

$$
\|u-\hat{u}\|_{C}=O(h)
$$


This theorem is a direct corollary of estimate (4.8).

Remark 3. It follows from estimate (4.10) that all solutions in $B$ to problem (1.2) belong to some ball of radius of order $h$.

\section{Conclusions}

Theorems 1-3 justify rigorously the closeness of the semi-discrete and continuous model problems describing the diffusion absorption process in the array of pointwise cells. It means that the semi-discrete problem can be homogenized. However, for real problems it may be preferable (from the modelling point of view) to use semi-discrete models with a reduced number of cells because the real quantity of cells is too large for numerical computations. Then Theorem 3 justifies this approach: the two semi-discrete models (with more cells and with less cells) will be close because they are both close to the continuous model.

Let us discuss some possible generalizations. The considered model is one-dimensional. In a three-dimensional setting the cells may be modeled by small spherical holes forming an $\varepsilon$-periodic cubic net with the diameter of the holes of order $\varepsilon^{3}$ which generates the so called "terme étrange" in the homogenized equation corresponding to a continuous absorption as in equation (1.4) (see [15], [9]).

\section{Acknowledgements}

The work was financially supported by the French-Russian PICS BIOMAT CNRS-RFBR grant.

\section{References}

[1] N.S. Bakhvalov, Averaging of partial differential equations with rapidly oscillating coefficients, Doklady Acad. Nauk SSSR 224 (2), pp. 351-355, 1975.

[2] A.L. Baldwin, G. Thurston. Mechanics of endothelial cell architecture and vascular permeability. Crit Rev Biomed Eng., 29 (2), pp. 247-78, 2001

[3] N. Bessonov, V. Volpert, Dynamic models of plant growth, Mathematics and Mathematical Modelling. Publibook, 2006.

[4] X. Blanc, C. Le Bris, P.L. Lions, From molecular models to continuum mechanics. Archive for Rational Machanics and Analysis, 164, 4, pp. 341-381, 2002.

[5] X. Blanc, C. Le Bris, F. Legoll, Analysis of a prototypical multiscale method coupling atomistic and continuum mechanics, ESAIM: M2AN, Vol. 39, n. 4, 797-826, 2005.

[6] M. Born, K. Huang, Dynamical Theory of Crystal Lattices, Oxford U. P., London, 1954. 
[7] A. Desmoulière, C. Chaponnier, G. Gabbiani. Tissue repair, contraction, and the myofibroblast. Wound Repair Regen., 13 (1), pp. 7-12, 2005.

[8] D. Caillerie, A. Mourad, A. Raoult, Discrete homogenization in graphene sheet modeling. Journal of Elasticity, vol. 84, pp. 33-68, 2006.

[9] D. Cioranescu, F. Murat, Un terme étrange venu dailleurs, I and II, Nonlinear partial differential equations and their applications, Collége de France seminar, Vol. 2 and 3, ed. by H.Brézis and J.L.Lions, Research notes in mathematics, 60 pp.98-138, and 70 pp.154178, Pitman, London (1982).

[10] L.C.Evans, Partial Differential Equations, Graduate Studies in Mathematics, V.19, AMS, Providence, 1998.

[11] L. He, X. Wang, H.L. Tang, D.J. Montell. Tissue elongation requires oscillating contractions of a basal actomyosin network. Nature Cell Biology 12, pp. 1133-1142, 2010.

[12] S.M. Kozlov, Averaging of difference schemes, Math. USSR Sb., Vol 57, pp. 351-369, 1987.

[13] I.A. Kunin, Elastic Media with Microstructure, I. Vol. 26, Springer-Verlag, Berlin, NewYork, 1982; II. Vol. 44, Springer-Verlag, Berlin, New-York, 1983.

[14] P.Kurbatova, G. Panasenko,V.Volpert, Asymptotic-numerical analysis of the diffusiondiscrete absorption equation, Math. Methods in the Applied Sciences, vol. 35, 2012, pp. 438-444 doi 10.1002/mma.1572

[15] V. Marchenko, E. Khruslov, Boundary value problems in domains with a fine grained boundary, (in Russian) Naukova Dumka, Kiev, 1974.

[16] R. Orive, E. Zuazua, Finite difference approximation of homogenization problems for elliptic equations, Multi-scale Methods and Simulation 4, 2005, pp. 36-87.

[17] G. Panasenko, Multi-scale Modeling for Structures and Composites, Springer, 2005.

[18] G. Panasenko, Partial homogenization: continuous and semi-discrete versions, Math. Models and Methods in Applied Sciences, 17, 8, 2007, pp. 1183-1209.

[19] V. Shenoy, R. Miller, E.B. Tadmor, D. Rodney, R. Philips, M. Ortiz, An adaptive finite element approach to atomistic-scale mechanics - The Quasi-Continuum Method, J. Mech. Phys. Solids 47, p. 611, 1999. 